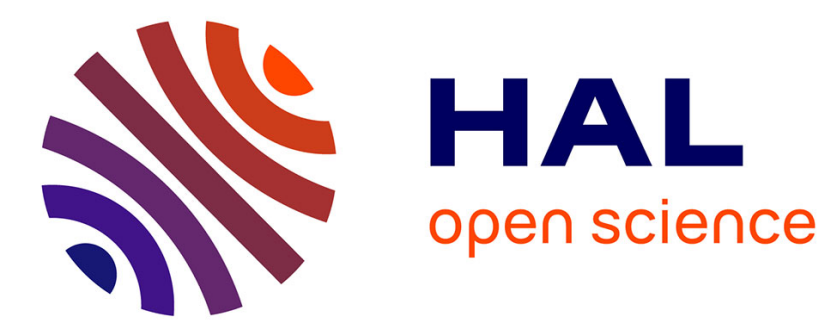

\title{
Les mutations du travail social: de la transformation du public aux changements dans les modes de prise en charge \\ Olivier Cousin
}

\section{To cite this version:}

Olivier Cousin. Les mutations du travail social: de la transformation du public aux changements dans les modes de prise en charge. Sociologie du Travail, 1996, 96 (2), pp.141-161. hal-02091734

\section{HAL Id: hal-02091734 \\ https://hal.science/hal-02091734}

Submitted on 6 Apr 2019

HAL is a multi-disciplinary open access archive for the deposit and dissemination of scientific research documents, whether they are published or not. The documents may come from teaching and research institutions in France or abroad, or from public or private research centers.
L'archive ouverte pluridisciplinaire HAL, est destinée au dépôt et à la diffusion de documents scientifiques de niveau recherche, publiés ou non, émanant des établissements d'enseignement et de recherche français ou étrangers, des laboratoires publics ou privés. 


\section{Olivier Cousin}

\section{Les mutations du travail social : de la transformation du public aux changements dans les modes de prise en charge *}

Les populations auxquelles sont confrontées les assistantes sociales ont changé : elles sont beaucoup plus diversifiées qu'auparavant, plus averties de leurs droits et présentent des types de problèmes plus divers. L'auteur montre que pour faire face à cette évolution, les assistantes sociales ont adopté de nouvelles formes d'organisation de leur travail et développé de nouveaux outils. Ces changements ont contribué à modifier en profondeur le sens même du travail social qui s'est éloigné du modèle psycho-éducatif pour rentrer dans la problématique de la réinsertion. À partir de cette analyse et de celle de la signification des contrats que passent les travailleurs sociaux avec les usagers, l'auteur révèle les ambiguïtés nées de cette évolution de l'action sociale.

A l'instar des grandes institutions, école, justice, hopital..., le travail social doit surmonter le défi d'un retour de la grande pauvreté en essayant de lier deux logiques : préserver son identité et faire preuve d'innovation. Tout changement est source de crise et de tension révélant la force et la faiblesse des acteurs. Mais au-delà d'une simple adaptation à une situation, il donne à voir les nouveaux modes d'identifications et de définition de soi des acteurs. C'est dans ce sens qu'il faut comprendre les innovations introduites par les travailleurs sociaux dans l'organisation de leur travail afin de faire face à un changement du public et à une transformation du cadre institutionnel.

Avec la persistance d'un chômage structurel qui ne cesse d'augmenter et l'émergence d'une population définie par l'exclusion sociale, les centres médicaux sociaux (CMS) gèrent des effectifs en augmentation constante. Et parce que le public des CMS connaît des parcours divers et très chaotiques, les travailleurs sociaux doivent aussi répondre à des demandes multiples couvrant des domaines très larges. De manière

\footnotetext{
* Cet article s'appuie sur une recheche menée en collaboration avec C. Dogant, sous la direction de F. Dubet. Elle a été réalisée à la demande du conseil Général, Direction Solidarité Gironde. Cf. (Cogant et Cousin, 1995).
} 


\section{Olivier Cousin}

concomitante à ce changement de public, les CMS se trouvent confrontés à un environnement institutionnel profondément transformé. Pour répondre aux problèmes urbains et à la précarité, les pouvoirs publics ont introduit, depuis le milieu des années quatre-vingt, dans le champ du travail social des nouveaux professionnels chargés d'appliquer des nouveaux dispositifs (RMI, politique de la ville...). À divers degrés les travailleurs sociaux, et plus particulièrement les assistantes sociales, sont directement concernés par ces mesures, ne serait-ce que parce qu'ils sont ceux qui connaissent le mieux les populations visées par ces dispositifs et qu'ils instruisent les dossiers pour obtenir des prises en charge.

Les nouveaux modes d'organisation des CMS correspondent à une rationalisation du travail social. Ils permettent aux assistantes sociales de maîtriser les flux, en contenant l'urgence des demandes des usagers, et ils inscrivent l'action des travailleurs sociaux dans un champ plus large, en harmonisant leur intervention avec les autres organismes sociaux. Mais plus généralement, en modifiant l'organisation des CMS, les assistantes sociales redéfinissent leur public et le travail social. Pour illustrer ces changements, nous nous appuierons sur une recherche conduite auprès de six centres médicaux sociaux de la Gironde, dont un a fait l'objet d'une attention particulière et soutenue, pendant un an. La recherche a commencé au moment où ce centre s'est engagé dans de nouvelles pratiques, nous avons donc pu suivre l'élaboration de la réforme et apprécier ses évolutions. Ces CMS sont des cas particuliers, mais les options choisies pour répondre aux nouveaux enjeux du travail social reflètent les réflexions et les expérimentations à l'œuvre dans d'autres départements. ${ }^{1}$ Nous analyserons les nouvelles contraintes des CMS, puis les grands axes traduisant les changements d'organisation. Enfin, nous essayerons de saisir quels sont les enjeux d'un tel changement pour le travail social.

\section{CHANGEMENT D'ORGANISATION ET TRANSFORMATION DE L'ENVIRONNEMENT}

\section{Un changement du public}

Tous les travailleurs sociaux constatent un accroissement massif de la population s'adressant à leur service du fait de l'augmentation des personnes touchées

\footnotetext{
1. A. Durrleman (mars 1993), (sous la direction de). La commission chargée de l'atelier "Evolution du travail social" pose les problème dans les mêmes termes que les CMS étudiés. Elle parle d'hétérogénéité du public, de l'émergence des nouveaux métiers, de redéfinir le diagnostic social...
} 
Les mutations du travail social par la pauvreté et la précarité. ${ }^{2}$ Mais, c'est surtout une très grande diversité du public que les assistantes sociales doivent gérer. Le chômage et la précarité n'épargnent plus aucune catégorie. Les jeunes sont particulièrement affectés, les études n'offrent plus la même garantie et ne protègent pas toujours contre le chômage ou les emplois précaires. Les moins diplômés sont les plus exposés mais les jeunes ayant un niveau d'instruction égal ou supérieur au niveau bac +2 ne sont pas épargnés. ${ }^{3}$

Les CMS reçoivent donc un public hétérogène où se croisent des jeunes avec un haut niveau de qualification qui, ne pouvant bénéficier du chômage, viennent chercher des aides sociales avant de trouver un véritable emploi ${ }^{4}$; des familles ne pouvant vivre et parfois survivre sans l'aide des assistantes sociales et n'ayant aucun espoir de trouver un emploi ; des personnes socialement intégrées qui ont besoin des travailleurs sociaux pour trouver un logement car leurs revenus sont trop faibles pour accéder au parc de logements privés ; ou encore, des familles socialement et psychologiquement trop démunies pour élever et éduquer leurs enfants sans l'aide d'un travailleur social. Les demandes et les réponses à apporter varient donc, obligeant les travailleurs sociaux à couvrir des domaines très vastes. Il n'y a plus un public type mais un éclatement du profil des usagers ayant des attentes à l'égard des travailleurs sociaux à la fois précises et diversifiées.

L'hétérogénéité du public s'inscrit aussi dans un contexte particulier, celui de «l'exclusion» qui place les travailleurs sociaux dans une situation plus complexe ne pouvant plus s'appuyer sur les réseaux traditionnels (l'entreprise et l'institution familiale, notamment). Ce qui change aujourd'hui, ce ne sont pas tant les problèmes de la pauvreté que le fait que ces problèmes ne s'inscrivent plus dans les rapports sociaux traditionnels. L'exclusion et la marginalité sont vécues et interprétées dans un vide social dans lequel les conflits sociaux n'y ont plus de place. Surtout, il n'y a plus de principe d'unité entre les différents problèmes. L'image d'un éclatement des problèmes est, de ce point de vue, plus juste que l'idée d'une crise. Car, en réalité, les «exclus» ne sont ni complètement «dedans» ni totalement «dehors». A l'image des jeunes Beurs,

\footnotetext{
2. 3,3 millions de personnes sont inscrites à l'ANPE en mars 1995, soit environ 13\% de la population active. Si l'on ajoute le nombre de personne occupant des emplois précaires ou bénéficiant des aides sociales (RMI, CES, TUC...) se sont environ cinq millions de personnes en âge de travailler qui gravitent autour du marché de l'emploi. Le nombre de chômeurs était de 1,9 million en 1981 et de 300.000 en 1970. Si le nombre de chômeurs baissent sensiblement depuis le début de l'année 1995, le nombre de chômeurs de longue durée ne cesse d'augmenter ( $+40 \%$ en deux ans), ainsi que celui des chômeurs en fin de droit $(+38 \%)$.

3 . le taux de chômage neufs mois après la fin des études est proche de $80 \%$ pour ceux qui n'ont aucun diplôme, il est proche de $60 \%$ pour ceux qui ont le niveau CAP - BEP, proche de $50 \%$ pour ceux qui ont le niveau bac et, enfin, proche de $25 \%$ pour ceux qui ont atteint un niveau bac plus deux et plus. « Bilan formation - emploi ». Economie et Statistique, n 277/278, février 1995.

4. Sur un des CMS où nous avons travaillé, situé à proximité d'un campus universitaire, $20 \%$ des bénéficiaires du RMI ont un niveau d'étude supérieur à bac +5 . Si cette situation est exceptionnelle, elle marque néanmoins l'éclatement du public se présentant dans les CMS.
} 


\section{Olivier Cousin}

nombreux sont ceux qui peuvent être définis par cette contradiction entre une assimilation culturelle forte et une faible intégration sociale. ${ }^{5}$ On parle alors d'une dualisation de la société, dualisation qui sépare les individus en fonction de leur degré d'intégration sociale mais qui traverse aussi de nombreuses personnes elles-mêmes. Les individus ne sont plus des personnages définis par des rôles et des statuts, au contraire, ils apparaissent comme des êtres fragiles et parfois contradictoires qui ne se donnent plus à lire dans leur totalité. ${ }^{6}$ Ce qui entraîne des changements d'attitude et de comportement vis à vis des assistantes sociales.

\section{Un changement d'approche et d'attitude}

La «clientèle» traditionnelle des centres sociaux était largement définie par deux ordres de problèmes interdépendants. Le premier concerne la précarité économique. Les usagers pris en charge par les travailleurs sociaux sont issus des milieux populaires à faible niveau de qualification, ils sont même assez souvent illettrés. ${ }^{7}$ Le deuxième aspect concerne l'insertion des usagers dans la société. Ils sont principalement définis comme des personnes inadaptées, parfois déficientes et pouvant représenter une menace pour le groupe. L'inadaptation est appréciée à partir d'un manque caractérisant la personne, nuisible à son intégration, ou celle de sa famille, dans le travail ou dans la communauté. Les usagers sont qualifiés par leur situation économique et par leur déficience d'ordre psychologique, relationnelle ou physique. Les travailleurs sociaux, par le biais de la relation, doivent donc apprendre aux «inadaptés» à maîtriser leur existence et à s'intégrer. Ils visent aussi à protéger la société des dangers de leurs comportements jugés irrationnels et de leur mauvaise socialisation, en proposant aux usagers un soutien moral et psychologique.

Si cette population subsiste, tout laisse croire, qu'aujourd'hui, elle n'est plus nécessairement majoritaire et, surtout, qu'elle peut être définie à partir de ces deux critères. Il n'y a plus aujourd'hui une population mais des populations dont la définition est complexe. C'est pourquoi, on ne peut plus parler de clientèle ou de catégorie, le pluriel s'impose comme une évidence, il y a dorénavant des publics cibles. ${ }^{8}$ En réalité, ce qui permet de qualifier le plus facilement le public des centres sociaux c'est moins des caractéristiques propres que les dispositifs mobilisés pour venir en aide aux usagers. Les problèmes se recoupent et les usagers viennent pour résoudre plusieurs difficultés souvent étroitement dépendantes mais, selon les actions dans le cadre du suivi ou selon les dispositifs proposés, les travailleurs sociaux recomposent des publics. On parle alors

\footnotetext{
5. Cf. G. Kepel, (1987). D. Lapeyronnie, (1993).

6 . F. Dubet, (1994).

7. Cf. J. Verdès-Leroux, (1978). Plus particulièrement le chapitre 4, « la cible du service social ».

8 . J. Ion, « La fin des petits clercs? », in J. Donzelot (sous la direction de) (1991). P. Rosanvallon, (1995).
} 
Les mutations du travail social des «R.M.istes», des familles ayant des problèmes de logements ou des ennuis de santé ou, encore, de celles qui ont des difficultés éducatives. Les critères pertinents pour qualifier ce nouveau public sont à la fois son parcours social et le dispositif de prise en charge qui marquent le degré d'engagement des travailleurs sociaux.

Les travailleurs sociaux observent un changement de comportement de la part des usagers. Les services sociaux sont, avant tout, des lieux de redistribution. Les usagers se déplacent dans les centres sociaux pour obtenir une information ou une aide matérielle diverse (aide financière ou demande d'allocation). Ils sont à la fois des consommateurs avertis, connaissant les dispositifs capables de subvenir à leur besoin, et dépendant des services sociaux pour obtenir un soutien et constituer un dossier. L'image d'un public totalement démuni poussant la porte des CMS et se laissant complètement prendre en charge, ne tient plus. Les usagers sont exigeants, réclament l'accès à une prise en charge quelquefois perçue comme un «dû» et sont capables de formuler une demande complexe. Ils deviennent des stratèges pouvant faire pression sur les travailleurs sociaux en invoquant l'urgence de leur demande quand ils comprennent que le simple exposé de leur problème n'est plus une garantie suffisante pour être reçu par une assistante sociale. Que ce soit à l'école, dans le secteur de la santé ou dans les services sociaux, les relations entre les individus et les institutions s'inscrivent dans un rapport instrumental, et les utilisateurs des services agissent comme des consommateurs. ${ }^{9}$

Ce nouveau rapport aux services sociaux transforme les liens entre les travailleurs sociaux et leurs «clients». Une grande partie des usagers souhaite mettre une distance entre l'entrée dans un dispositif et la relation avec l'assistante sociale. Ils ne désirent pas un service déshumanisé, sorte de guichet délivrant des informations ou de l'argent, mais la relation telle qu'elle est entendue dans le sens traditionnel du travail social doit être partiellement revue. Tant que le travail social s'inscrivait dans une société intégrée, il était possible d'aborder le problème des usagers en termes de déficit de socialisation. La nécessité pour les travailleurs sociaux était alors de proposer à la fois des aides matérielles et un modèle d'insertion, afin de remettre les individus déviants sur la bonne voie. Or, aujourd'hui, quelques-uns refusent cette approche parce qu'ils ne se définissent pas comme marginaux par vocation ou constitution, mais comme précarisés par l'effet de la crise de l'emploi et par la faute de la société. ${ }^{10}$ Le prix de la prise en charge soumise à la relation psychologique peut paraître trop lourd et surtout ne pas se justifier. Pour avoir accès à un logement ou pour bénéficier d'une aide financière, les usagers ne sont pas prêts à livrer leurs affects.

\section{Les nouvelles contraintes de l'environnement}

9. Cf. R. Ballion, (1982).

10. J. Donzelot, « Le social du troisième type », in J. Donzelot op. cit. 


\section{Olivier Cousin}

Parallèlement à la pression des usagers et à leur changement de comportement, les assistantes sociales évoluent aussi dans un contexte en mutation. Face à la montée de la pauvreté et aux problèmes urbains une multitude de nouveaux dispositifs ont été créés. Ces dispositifs relèvent de la compétence des travailleurs sociaux et sont animés ou conduits par de nombreux partenaires. Ils engendrent donc un surcroît de travail et exigent de nouvelles compétences car ils demandent un haut degré de technicité.

Ces partenaires n'ont bien souvent pas la même culture que les travailleurs sociaux et n'utilisent pas les mêmes outils méthodologiques. ${ }^{11}$ Ils imposent un nouveau modèle d'intervention dans le champ social qui ne se base plus sur la relation et le soutien psycho-éducatif mais sur des critères d'efficacité. La concertation avec ces nouveaux partenaires devient une nécessité pour les travailleurs sociaux au risque de voir s'échapper une partie de la maîtrise de l'action sociale. Sauf à refuser les nouveaux dispositifs d'insertion, les travailleurs sociaux ne peuvent plus intervenir seuls auprès des familles. Le RMI en est l'exemple presque parfait, mais ceci est aussi vrai pour les problèmes d'accès au logement.

L'arrivée des nouveaux dispositifs oblige donc les assistantes sociales à repenser leurs modes de prise en charge des usagers. Le travail social devient de plus en plus un travail d'équipe dans lequel l'information doit circuler et les dossiers s'échanger. Ceci, d'autant plus que la «clientèle» change et qu'une nouvelle population s'adresse aux CMS. C'est particulièrement vrai pour les personnes dites «isolées» qui arrivent en masse dans les services sociaux. Or, cette population était inconnue des centres, ce qui signifie que les dispositifs prévus à son effet restent encore peu connu de la polyvalence ${ }^{12}$. Les assistantes sociales doivent donc nécessairement s'ouvrir à d'autres partenaires afin de répondre au changement.

\section{LES NOUVEAUX MODES D'ORGANISATION DES CENTRES MÉDICAUX SOCIAUX}

Pour répondre à l'augmentation du public s'adressant aux CMS, à la pression exercée par les usagers sur les travailleurs sociaux et ne pas s'isoler des nouveaux partenaires, les six centres étudiés ont opté pour des changements similaires. Les CMS

\footnotetext{
11. Cf. I. Astier, «Chronique d'une commission locale d'insertion», in J. Donzelot op. cit. J. Ion, (1990). 12. « $50 \%$ des bénéficiaires du RMI étaient inconnus des services sociaux de secteur », ce qui souligne le caractère familial des services polyvalents, une forte proportion des RMistes vivant seuls n'appartiennent pas aux publics cibles antérieurement visés. Rapport de la Commission Nationale d'évaluation du RMI,(1992), Paris, La Documentation française.
} 
Les mutations du travail social se sont vu accorder le droit d'une nouvelle organisation par leur organisme de tutelle, mais les choix d'organisation sont de leur propre initiative. Les changements cherchent à rationaliser le travail, à améliorer l'accueil et la réception du public et à réduire l'isolement des assistantes sociales. Parfois les moyens utilisés divergent selon les centres mais l'esprit de ce changement est le même. Quatre modes d'organisation retiennent particulièrement l'attention : l'instauration ou le renforcement d'un pôle d'accueil ; la volonté de mettre fin à la sectorisation ; la tentation d'introduire de la spécialisation dans la polyvalence ; l'instauration de contrat avec les usagers.

\section{L'accueil du public}

Dans l'ensemble des centres étudiés, le problème de l'accueil des usagers se pose. Avant les réformes de structure, le public venait sans rendez-vous et rencontrait directement une assistante sociale. Devant l'afflux des personnes, ce fonctionnement devient impossible. Les assistantes sociales ne peuvent recevoir immédiatement tout le monde et les temps d'attente s'allongent rapidement. Pour tenter de contourner ce problème, les usagers invoquent l'urgence de leur cas. Rapidement un cycle infernal s'installe et c'est l'ensemble des CMS qui est submergé par les demandes. Pour mettre fin à cette situation les CMS instaurent un principe d'accueil inspiré du modèle hospitalier. L'accueil s'impose, autant pour rationaliser le travail social que pour contrer les stratégies des usagers. Selon les centres, les usagers rencontrent en premier lieu une secrétaire médico-sociale et/ou une assistante sociale spécialement affectée à l'accueil. Ces professionnelles se chargent d'enregistrer la «demande» des personnes et proposent plusieurs solutions. Soit elles orientent les usagers vers d'autres organismes sociaux qui peuvent les prendre en charge. Soit elles répondent immédiatement à la «demande» quand il s'agit de donner des informations ou de fournir des dossiers. Soit elles proposent un rendez-vous avec une assistante sociale.

Dans les faits, l'accueil remplit d'abord une fonction d'information et d'orientation permettant de désengorger les CMS. En effet, les usagers ont la garantie d'être immédiatement reçus et leur dossier, d'être immédiatement enregistré. C'est ainsi que dans un CMS, sur une période de cinq semaines, 222 personnes différentes se sont présentées à l'accueil. Parmi celles-ci, 42\% sont orientées vers d'autres organismes sociaux et, parmi celles qui relèvent du CMS, 50\% en restent au stade de l'accueil. L'accueil permet donc aux CMS de «filtrer» le public en fonction de la nature de la «demande» faisant ainsi tomber la notion d'urgence. Les travailleurs sociaux chargés des prises en charge des usagers, c'est à dire ceux qui mettront en place des dispositifs particuliers afin de répondre à des problèmes précis, se voient ainsi libérés des fonctions d'information et d'orientation. L'accueil modifie aussi de manière plus profonde les 
Olivier Cousin

CMS. Il les transforme en service car il nécessite une collaboration étroite entre les professionnels chargés d'enregistrer les demandes et ceux qui élaborent des prises en charge. Les travailleurs sociaux s'échangent ainsi les dossiers et engagent une action à partir du diagnostic élaboré par les professionnels de l'accueil. Pour que l'accueil fonctionne correctement il est donc nécessaire que les centres définissent collectivement les fonctions et les limites de l'accueil et les moments où les assistantes sociales chargées d'organiser un suivi social prennent le relais.

\section{La remise en cause de la sectorisation}

Le fonctionnement traditionnel des CMS attribue à chaque assistante sociale un secteur particulier ${ }^{13}$ sur lequel elle intervient seule. Ce secteur peut ne couvrir qu'un îlot, s'étendre à un quartier ou encore englober la quasi-totalité d'une commune selon la taille des circonscriptions. Ce découpage n'est plus satisfaisant aujourd'hui. D'abord parce qu'il repose sur un isolement des assistantes sociales, seules en charge et responsables d'un secteur. Il nuit donc à tout travail collectif au sein des CMS. Ensuite, parce qu'il ne permet pas aux travailleurs sociaux d'harmoniser leur intervention avec les politiques urbaines qui couvrent des espaces beaucoup plus vastes que les secteurs. Les travailleurs sociaux tentent donc de dé-sectoriser leur champ d'intervention, soit en regroupant plusieurs secteurs, soit en intervenant indifféremment sur l'ensemble de la commune.

La dé-sectorisation offre l'avantage de briser l'isolement des travailleurs sociaux en leur donnant la possibilité de travailler de manière plus étroite avec d'autres partenaires. Ce nouveau découpage permet surtout aux travailleurs sociaux de ne pas s'exclure des nouvelles politiques sociales et de tisser des relations de partenariat indispensables pour gérer les nouveaux dispositifs. Enfin, la dé-sectorisation donne aussi la possibilité d'une meilleure répartition des suivis. En effet, les assistantes sociales ne sont plus nécessairement tenues de prendre en charge une famille, ou une personne, parce qu'elle habite sur un secteur donné. La dé-sectorisation permet de se répartir les dossiers en fonction de la disponibilité de chacune et évite les trop grandes disparités entre les professionnelles comme c'était le cas auparavant. Ce changement renforce donc la notion de service. Les usagers s'adressent moins à une assistante sociale qu'à un service capable de leur répondre en fonction de la disponibilité de ses membres.

\section{Les contraintes de la polyvalence}

\footnotetext{
13. Un secteur ne correspond pas à une réalité géographique, il est défini par rapport à une densité de population. Généralement un secteur correspond à une population de 5.000 habitants.
} 
La polyvalence est une des notions clé du travail social. Les assistantes sociales employées dans les CMS interviennent dans tous les domaines de l'action sociale. Or la multiplicité des dispositifs et leur complexité rend parfois difficile la possibilité de maintenir le principe de la polyvalence. C'est pourquoi, dans certains centres, les travailleurs sociaux tentent d'introduire une spécialisation. Selon le contexte, ils choisissent de prendre en charge des populations particulières, par exemple les personnes vivant seuls, ou ils choisissent de se spécialiser dans la maîtrise de certains dispositifs, par exemple le RMI. Il ne s'agit ici que d'orientation car en réalité les travailleurs sociaux restent attachés à la notion de polyvalence et ne veulent pas véritablement se spécialiser. À cette volonté de préserver une part de la polyvalence s'ajoutent les réticences de la direction départementale qui se méfie de la spécialisation. Il faut donc à ce propos parler de spécialisation partielle.

La quasi-spécialisation permet d'abord aux professionnelles d'être plus efficaces. En choisissant de travailler en priorité avec un type de population ou sur un dispositif, les assistantes sociales peuvent multiplier les réseaux avec les professionnels intervenant sur ces domaines. Elles améliorent ainsi l'efficacité de leurs prises en charge. La quasi-spécialisation est donc un moyen pour les travailleurs sociaux de s'inscrire dans des actions collectives et de s'ouvrir à d'autres partenaires sociaux. Elle modifie aussi les équilibres au sein des CMS. En effet, la spécialisation partielle permet les échanges d'informations et, quelquefois, de sous-traiter les problèmes. Ainsi, dans un des CMS, trois assistantes sociales travaillent sur le même territoire et chacune s'oriente plus particulièrement dans un domaine (RMI, logement et enfance). Quand l'une d'entre elles prend en charge une famille, si un des volets de l'intervention concerne le logement, sa collègue «spécialisée» dans ce domaine gérera ce volet sans qu'elle intervienne directement auprès de la famille. Bien que l'échange d'informations et de services se fasse entre professionnelles, la confidentialité, pilier de la notion du colloque singulier, vole ici en éclat.

\section{Le «contrat», une nouvelle norme de travail}

Dans la vision traditionnelle du travail social, le suivi et les prises en charge des personnes reposent sur une relation étroite entre l'assistante sociale et l'usager. Le travail s'élabore au cour de cette relation et l'«accompagnement» relève de la seule responsabilité de l'assistante sociale. Pour les situations les plus délicates, comme les problèmes concernant les enfants, le suivi social peut s'inscrire dans une durée quasi illimitée. Cette logique se justifie tant que l'orientation du travail social cherche à combler des déficiences, mais quand il s'agit de palier aux problèmes de l'insertion et du 
Olivier Cousin

chômage elle devient extrêmement lourde, voire handicapante. C'est pourquoi les travailleurs sociaux ont introduit deux changements majeurs. L'essentielle des actions entre dorénavant dans une logique d'évaluation, les travailleurs sociaux agissent par objectifs et soumettent leurs actions à des critères d'évaluation. Ainsi, c'est moins une personne qui est prise en charge que des problèmes que l'on cherche à résoudre. Ce qui conduit à un deuxième changement : les suivis s'inscrivent dans une durée délimitée. Les assistantes sociales proposent un calendrier soumis à l'évaluation. Ce changement est particulièrement important puisqu'il signifie formellement aux usagers qu'un suivi prend fin une fois les objectifs fixés atteints. À ce propos les travailleurs sociaux parlent de contrat.

En introduisant l'idée de contrat et en soumettant les prises en charge à un principe d'évaluation, les travailleurs sociaux veulent sortir de la logique de la dépendance et de l'assistanat. Le contrat introduit un changement à la fois symbolique et réel car il bouleverse la représentation du travail social. Dans cette logique les travailleurs sociaux cherchent à faire des usagers des acteurs de leur propre prise en charge en les faisant participer de manière plus active. Par ailleurs, l'instauration d'un contrat est un moyen de clarifier et de rationaliser leurs actions. En imposant une durée à la prise en charge les travailleurs sociaux veulent rompre avec l'image étouffante de l'assistanat. Pour marquer cette rupture, dans certains centres, les travailleurs sociaux ont changé les modes d'attribution des dossiers. Profitant de la dé-sectorisation, les assistantes sociales ont échangé leurs dossiers. Contrairement aux attentes, les usagers ont dans l'ensemble accepté ce changement mettant en avant la compétence professionnelle des assistantes sociales plutôt que la personnalisation de la prise en charge. «Je ne suis pas indifférente à l'assistante sociale avec qui je travaille, mais ce n'est un problème de changer», déclare une personne suivie depuis douze ans. Pour leur part, les assistantes sociales ne se sentent plus systématiquement responsables des personnes vivant de et par l'aide sociale comme l'explique l'une d'entre elles : « Les contrats nous rendent uniquement responsables des gens avec lesquels on a passé un contrat. Donc on n'est pas censé s'occuper de tout ». Enfin, la contractualisation renforce, là aussi, le travail d'équipe. Les décisions de prise en charge et l'évaluation des actions se font souvent dans le cadre des réunions d'équipe qui fixent les objectifs et la durée du suivi.

La notion de contrat, proposée par les équipes dans le cadre des suivis, doit être fortement nuancée. Pour les usagers elle a peu de sens car, pour les personnes les plus démunies socialement et vivant quasi exclusivement des aides sociales, la possibilité de sortir des dispositifs est rarement envisageable. La notion de contrat est d'autant plus floue pour les usagers qu'ils ne viennent pratiquement jamais au CMS pour rendre compte des démarches qu'ils ont pu faire afin de résoudre leur problème. De ce point de 
Les mutations du travail social vue, l'image des usagers devenant les acteurs de leur propre prise en charge est toute relative. Le contrat doit être compris différemment. C'est un outil de travail et non un véritable contrat dans la mesure où un contrat se passe entre égaux et où les usagers n'ont guère à offrir dans le contrat. Le contrat se présente comme un programme et une alliance entre les usagers et un service afin de réaliser des objectifs précis. Il inscrit l'action dans le temps et donne du sens à la relation rompant ainsi avec la dépendance qui menace et alourdit toute situation d'aide. Il permet de clarifier les situations et offre la possibilité aux travailleurs sociaux, comme aux usagers, de repenser les prises en charge. Pour les usagers, par exemple, il permet de hiérarchiser les problèmes. Ainsi, quand ils se présentent, ils dissocient leur expérience des prises en charge conduites par les assistantes sociales. Une mère de famille raconte son parcours : « J'ai trois enfants, je ne travaille pas et je les élève seule. Je viens pour demander des aides, pour avoir des stages et faire garder mes enfants. Je veux me séparer de mon concubin, ça ne marche pas. Je veux aussi trouver un autre appartement. Avec l'assistante sociale on règle chaque problème à la fois. Là, on essaye d'avoir une aide RMI ». En effet, ce sont moins des personnes que des problèmes qui sont traités dans le cadre des suivis, déchargeant ainsi les professionnelles et leur «clientèle» d'un sentiment de culpabilité car ce ne sont plus des individus qu'on évalue mais des objectifs et des dispositifs. Mais l'instauration d'un «contrat» ne va pas sans le risque d'une gestion bureaucratique des problèmes puisque au nom de l'efficacité, des familles peuvent être exclues de certains dispositifs. Si elles ne jouent pas le jeu, si elles ne respectent pas les contraintes imposées par les CMS, les assistantes sociales peuvent mettre fin à une prise en charge. Les familles ne sont pas pour autant exclues des CMS.

\section{Une rationalisation du travail}

Pour les travailleurs sociaux, comme pour leurs partenaires, la restructuration des centres procède principalement d'une rationalisation du travail. En organisant une structure d'accueil, en sortant du cadre restreint du secteur et en travaillant par objectif, les professionnels travaillent mieux et plus car l'urgence ne commande plus, et parce qu'ils accroissent la collaboration entre les travailleurs sociaux. Par exemple, pour un des CMS étudiés, la comparaison entre l'activité des assistantes sociales avant la restructuration (1993) et l'activité un an après indique qu'elles ont, pour une charge de travail équivalente, reçu plus d'usagers, fait plus de visites à domicile et participé à plus de réunions d'équipe. ${ }^{14}$ Elles gagnent un confort professionnel évident car un certain nombre de cas est immédiatement transféré vers d'autres services ou limité à des

14. En 1993, sur une période de deux mois (mars et juin), les douze assistantes sociales du CMS réalisaient 634 actes de travail et 801 en 1994 sur la même période. 
Olivier Cousin

dossiers administratifs. Le sentiment dominant est que l'impression de surcharge et de stress diminue. Les structures d'accueil remplissent donc leur rôle et elles offrent aux secrétaires médico-sociales une revalorisation de leur fonction en les plaçant au cœur du dispositif.

Pour les partenaires, les changements d'organisation, notamment les structures d'accueil et la dé-sectorisation, permettent de clarifier la situation et offrent la possibilité d'harmoniser leur champ d'intervention avec celui des CMS. La centralisation de l'information facilite la communication entre les différents services et diminue les actions redondantes. C'est particulièrement vrai avec les Centres Communaux d'Action Sociale (C.C.A.S). Pour les partenaires, comme pour les usagers, les assistantes sociales sont plus accessibles et plus disponibles, ce qui accroît encore la possibilité d'établir des actions communes. Par ailleurs, les partenaires des organismes sociaux très spécialisés (en particulier ceux qui interviennent dans le domaine du RMI ou du logement) sont assez défavorables à la spécialisation des assistantes sociales. Ils estiment que cette démarche n'est pas nécessaire et qu'elle couperait les assistantes sociales de leurs réseaux. Ils craignent aussi que l'information ne circule plus et qu'ils n'aient plus qu'un seul interlocuteur avec qui travailler. Comme les assistantes sociales, ils sont favorables à une spécialisation partielle. Ils défendent donc d'abord le principe de la polyvalence.

Mais ce changement d'organisation ne se limite pas à une amélioration des conditions de travail, il implique un bouleversement dans les modes de gestion des prises en charge. La notion de service s'impose. L'accueil des usagers, la désectorisation, la quasi-spécialisation et le travail par objectifs obligent les travailleurs sociaux à collaborer de manière plus étroite, à échanger leurs dossiers et leurs informations. Si le suivi social s'organise toujours autour d'une relation entre une personne et une assistante sociale, relation basée sur la confiance et, parfois, un attachement fort, les assistantes sociales travaillent de moins en moins seules. Les décisions et les objectifs des prises en charge s'élaborent dans le cadre des réunions d'équipe. Ainsi la responsabilité de l'action sociale dépend à la fois des assistantes sociales et du service dans lequel elles interviennent.

\section{UNE REDÉFINITION DE L'ACTION SOCIALE ET DES USAGERS}

Si la massification du public dépendant des CMS a contraint les travailleurs sociaux à s'engager dans des réformes de structure, les changements d'organisation, en retour, modifient les liens entre les assistantes sociales et leurs «clientèles». Ces 
Les mutations du travail social changements marquent la remise en cause du modèle traditionnel de l'action sociale. Le travail social ne s'inscrit plus dans une logique d'assistanat, au contraire, il est dominé par la problématique de «l'exclusion sociale» à la quelle les assistantes sociales tentent de répondre en diversifiant les modes de prise en charge.

\section{La crise du modèle traditionnel}

Le travail social en polyvalence de secteur s'appuyait sur quatre grands principes : une approche globale de la personne dans son fonctionnement individuel et social ; la priorité à des professions généralistes en milieu ouvert; une sectorisation de l'organisation pour garantir la proximité ; enfin, une mission globale d'animation donnée à l'action sociale. ${ }^{15}$ Or, ce modèle apparaît assez largement en crise, compte tenu de la diversité du public et de l'arrivée de nouveaux dispositifs. Le travail social devient trop complexe et les dispositifs font intervenir trop de professionnels pour que les assistantes sociales maintiennent ces principes.

Mais, ce sont surtout les supports professionnels qui se fragilisent le plus. Les assistantes sociales, en effet, peuvent de plus en plus difficilement se réclamer d'un modèle libéral et psycho-éducatif. Le modèle libéral signifie une relative autonomie professionnelle pour les travailleurs sociaux trouvant sa source dans le colloque singulier, la confidentialité et le secret. L'évaluation des prises en charge et le contrôle des actions n'y ont pas de place. Or, ce modèle entre en conflit avec l'extension du partenariat et l'institutionnalisation des contrats. Les travailleurs sociaux appliquent des règles d'efficacité qui remettent en cause le principe libéral. Il en va de même pour le modèle psycho-éducatif dont le principe est un travail sur une longue durée afin de " compenser des inégalités dues à des handicaps ». Le travail entrepris par les travailleurs sociaux visait à transformer les individus sans que les effets puissent s'évaluer. Il privilégiait la parole plutôt que l'affrontement à des situations réelles. Or, dans le cadre de l'exclusion du travail, cette dimension individuelle et réparatrice n'est plus adaptée, parce que les usagers la contestent et parce que les nouveaux dispositifs disqualifient ce modèle en lui en imposant un autre, de type contractuel. La notion de projet et le travail par objectif s'imposent et l'évaluation devient un outil indispensable.

\section{La diversité des prises en charge}

Pour faire face à ces nouvelles contraintes, les travailleurs sociaux modifient leurs modes de prises en charge. Ils se sont adaptés au nouveau contexte en diversifiant les degrés d'engagement auprès des usagers. À l'éclatement du public correspond un

15. Cf. J. Verdès-Leroux, op. cit., A. Durrleman, op. cit. 
Olivier Cousin

redécoupage des modes d'interventions. Les travailleurs sociaux inscrivent leurs actions dans un ensemble plus vaste touchant à la fois les problèmes de la ville et les politiques sociales, ce qui conduit à changer le regard sur les usagers. La notion de «public-cible» se substitue à la notion de «client», indiquant par là que les dispositifs ne s'adressent plus à des individus isolés mais à des populations pour lesquelles les différents intervenants tentent de reconstruire un tissu social à travers les mesures d'insertion. Pour J. Donzelot, cette transformation conduit à « un social du troisième type » défini par une nouvelle méthodologie de l'action sociale. ${ }^{16}$

Pour gérer ces nouvelles contraintes les travailleurs sociaux ont recours à de nouvelles définitions du travail social qui peuvent être caractérisées par un découpage des prises en charge en fonction du degré d'engagement des travailleurs sociaux, opposant les interventions «ponctuelles» aux interventions «globales». ${ }^{17}$ Les interventions «ponctuelles» désignent les actions dans lesquelles l'engagement des travailleurs sociaux dans la relation n'est pas l'essentiel de la prise en charge. Elles représentent l'essentiel de l'action des travailleurs sociaux. C'est ainsi que parmi les deux cent vingt-deux personnes s'étant présentées à l'accueil d'un CMS, 12\% ont eu un autre rendez-vous pour compléter définitivement leur dossier et $10 \%$ seulement ont fait l'objet d'un suivi social. Plusieurs actions relèvent de ce modèle. Tout d'abord, la réception dans le cadre des structures d'accueil. Les travailleurs sociaux diffusent de l'information, orientent éventuellement les usagers vers d'autres organismes ou enclenchent des dispositifs particuliers. Ici, les travailleurs sociaux sont essentiellement des techniciens du social capables de mobiliser des ressources. Ensuite, dans le cadre des suivis, l'action des travailleurs sociaux peut se limiter à l'évaluation d'une situation ou à la reconduction d'un mode particulier de prise en charge. Ils remplissent dans ce cas une fonction d'expertise visant à cautionner et à justifier une des décisions antérieures. C'est par exemple le cas lors de l'évaluation des dossiers pour l'agrément des assistantes maternelles. Enfin, toujours dans le cadre des suivis, les travailleurs sociaux, après l'élaboration d'un diagnostic social, gèrent des actions particulières dans lesquelles ils ne se trouvent pas nécessairement engagés. C'est, par exemple, le cas quand d'autres organismes prennent le relais. De ce fait le lien avec l'assistante sociale devient plus lâche. Son action consiste à mobiliser des partenaires et à harmoniser les pratiques. Le travailleur social n'est qu'un des interlocuteurs de l'usager. Dans le cas des interventions ponctuelles, les assistantes sociales rencontrent, en moyenne, entre deux et six fois par mois les personnes en suivi.

\footnotetext{
16. J. Donzelot, « Le social du troisième type », in J. Donzelot op. cit.

17. Une extrapolation sur année des personnes venant dans un CMS donne la répartition suivante : sur 2600 personnes pouvant se présenter à l'accueil, entre 260 et 540 feront l'objet d'un suivi social. Cette projection montre bien les changements dans le travail social et la part que représentent les prises en charge ponctuelles.
} 
Les mutations du travail social

L'intervention «globale» renvoie à la fonction plus traditionnelle de l'action sociale et fait directement référence à «l'action sociale globale». Elle s'appuie principalement sur le principe de la relation et engage très fortement le travailleur social dans la prise en charge. Elle se caractérise aussi par la durée, plus longue que dans le cas précédent et qui ne peut pas toujours être définie à l'avance. Les problèmes liés aux enfants correspondent parfaitement bien à ce type d'interventions ainsi que les signalements concernant la détresse psychologique des personnes ou les problèmes de maltraitante. Si les modes de prises en charge s'inscrivent toujours dans le même cadre, associant un travail psychologique en collaboration plus ou moins étroite avec des instances médicales et/ou un travail éducatif, en revanche, ce qui change c'est le critère de référence des travailleurs sociaux. Encore une fois, l'intention majeure de la prise en charge n'est plus sous-tendue par l'idée réparatrice mais par la problématique de l'insertion. $\mathrm{Ou}$, pour le dire autrement, c'est moins l'idée qu'il faut défendre la société contre les risques que lui font encourir certains individus, du fait même de leur déficience, que l'idée inverse, c'est à dire protéger l'individu contre les risques de la société et en particulier la «désaffiliation sociale». ${ }^{18}$ Ces deux volets de l'action sociale sont interdépendants mais, compte tenu de la dégradation des liens sociaux et de l'accroissement de la précarité, le souci de l'insertion l'emporte même si les assistantes sociales savent que les dispositifs n'ont qu'un faible impact. L'intervention globale offre alors un autre visage. La relation est peut-être moins un outil indispensable à l'élaboration d'un diagnostic et la condition de la réussite de l'action sociale, qu'un espace offert aux personnes afin de rompre avec la déshumanisation des relations sociales. ${ }^{19}$ Cette tendance n'est certes pas la fonction principale des travailleurs sociaux, que l'on ne peut assimiler à de simples confidents, mais elle marque bien la volonté de ne pas être uniquement des techniciens obsédés par l'efficacité. La difficulté est bien là, gérer ces deux axes parfois contradictoires.

\section{La notion de contrat}

Un autre aspect du changement dans les prises en charge concerne le contrat passé entre les travailleurs sociaux et les usagers. Le contrat est aujourd'hui au cœur de l'action sociale. Les nouveaux dispositifs s'accompagnent d'un contrat entre les usagers bénéficiaires d'une mesure et les organismes sociaux. Mais la notion de contrat recouvre des sens différents selon les interlocuteurs. Pour les travailleurs sociaux, il marque

18. R. Castel, « De l'indigence à l'exclusion : la désaffiliation », in J. Donzelot, op. cit. Cf. aussi R. Castel,(1995),. P. Rosanvallon, op. cit.

19. Pour les actions globales les assistantes sociales rencontrent, en moyenne, plus de sept fois la personne en suivi social. 


\section{Olivier Cousin}

l'inscription d'une prise en charge dans le temps, il signifie que le suivi est surtout une méthode de résolution des problèmes et non plus uniquement un soutien psychoéducatif. Il peut donc à ce titre prendre fin selon diverses modalités. Pour les usagers le contrat désigne principalement l'ordre de priorité des objectifs à atteindre dans le cadre d'un suivi. Il marque donc des étapes, mais il est rarement perçu comme un pacte. L'idée d'une rupture du contrat n'est pratiquement jamais envisageable. Si les interprétations divergent, le contrat n'en marque pas moins un changement profond pour l'action sociale. Il est la traduction d'une dette sociale de la société envers les plus démunis et, surtout, il incarne un changement dans la logique de l'Etat-providence. ${ }^{20}$

En effet, le contrat tel qu'il est passé avec les usagers devient un garant de la cohésion social parce qu'il représente un nouveau type de droit social. Car le droit à l'insertion est un droit accessible à tous qui signifie que les exclus sont fondés à obtenir un minimum de ressource. Le contrat engage le bénéficiaire dans une demande d'insertion et s'appuie sur une recherche de résultats. Il casse ainsi l'image du mauvais pauvre ou de l'assisté en inscrivant la personne dans un projet qui engage la double responsabilité de l'allocataire et de la communauté et doit déboucher sur la réinscription du bénéficiaire dans le régime commun. Pour P. Rosanvallon, il s'apparente à un «droit procédural» car c'est l'incidence sociale des comportements individuels qui est visée et non la rectification morale. ${ }^{21}$

Mais, dans la pratique et du fait même de la recherche de résultat, la notion de contrat comporte aussi quelques dérives, au moins dans son interprétation et dans son application. Tout d'abord, il existe une très grande diversité des contrats passés pour le R.M.I. Dans le cadre des commissions locales d'insertion (C.L.I), les projets d'insertion proposés aux personnes couvrent des réalités très différentes. Ils vont du «projet-santé» (inciter la personne à se soigner) au «projet professionnel». La nature des projets varie selon le profil social de la personne et de la durée du contrat. ${ }^{22}$ Les C.L.I sont le théâtre de rivalités entre les divers participants qui ont des lectures différentes des problèmes et des moyens à mettre en œuvre pour les résoudre. Elles sont également la scène où se définit la notion même d'insertion. Ce qui, au bout du compte, peut remettre en cause son principe, c'est à dire la prise en compte des spécificités de la personne dans une situation sociale donnée. Ce que des auteurs comme Rosanvallon et Castel présentent comme une obligation positive peut rapidement s'inverser et devenir une forme de discrimination ou prendre un caractère tautologique : seuls ceux dont on pense qu'ils peuvent s'insérer auront le droit à un vrai contrat d'insertion. L'idée même «d'inadapté» ou de «handicapé» menace donc constamment de refaire surface. Si l'on peut

\footnotetext{
${ }^{20}$. P. Rosanvallon, op. cit.

21. Ibid.

22. I. Astier, in J. Donzelot, op. cit., p. 77.
} 
Les mutations du travail social comprendre les réticences des travailleurs sociaux et des autres membres des C.L.I à l'égard du R.M.I, compte-tenu des faibles taux d'insertion économique (on estime à $15 \%$ les allocataires ayant trouvé un emploi stable ou précaire à la sortie du dispositif ${ }^{23}$ ) et des lourdeurs que représente un tel dispositif, il n'est pas certain que cette diversité des projets correspond à l'esprit du dispositif surtout lorsque d'un CMS à l'autre le nombre de projet d'insertion varie considérablement, autant pour des raisons «idéologiques» que techniques. ${ }^{24}$

Une autre dérive possible dans l'instauration du contrat concerne l'appréciation portée par les travailleurs sociaux sur les usagers eux-mêmes, que ce soit dans le cadre du R.M.I ou pour l'élaboration d'un suivi. Le contrat part du principe d'un engagement de la personne dans un processus particulier, impliquant l'adhésion à un projet. Il y a donc de la part du travailleur social une évaluation du comportement de la personne, voire un jugement. On voit ici la dérive à laquelle peut conduire cette évaluation puisqu'elle introduit l'idée de mérite ; idée qui se conjugue de différentes façons. Elle concerne en premier lieu, la capacité des personnes à respecter un calendrier. Elle peut aussi être invoquée lorsque les assistantes sociales prennent en charge les cas dits «lourds». Les travailleurs sociaux estiment parfois qu'il ne sert à rien de proposer des dispositifs d'insertion à ces personnes tant leur situation paraît insurmontable. Dans le cas du R.M.I, par exemple, cette catégorie d'individus ne disposera que du revenu minimum mais sera exclue du volet insertion. Enfin, l'idée de mérite est aussi un moyen de justifier l'absence de prise en charge quand les travailleurs sociaux sont surchargés de travail. Ici, le mérite se télescope avec l'idée de quota. Ne pouvant prendre en charge tout le monde et ne pouvant supporter «toute la misère du monde», les assistantes sociales vont sélectionner la population au nom de l'impossibilité de travailler avec telle ou telle personne. Si dans notre enquête nous n'avons jamais réellement constaté une telle dérive, nous avons néanmoins entendu ces propos lors des discussions avec les équipes des CMS. ${ }^{25}$

Ce qui est discutable, ce n'est pas que les travailleurs sociaux puissent faire des choix ou estiment que certains dispositifs sont inefficaces pour des personnes précises, c'est que ces choix paraissent souvent obscurs et ne font pas toujours l'objet d'une réflexion politique. Ce ne sont pas les travailleurs sociaux qui doivent opérer ces choix

\footnotetext{
23. Le RMI à l'épreuve des faits,(1991), Paris, Syros.

24. Dans un des centres étudiés, moins de $20 \%$ des persones bénéfiants du RMI s'inscrivent dans un projet d'insertion, alors qu'ailleurs ce sont plus de 50\%. Dans ce centre, les assistantes sociales, avec le soutien de la responsable de circonscription, élaborent peu de contrats parce qu'elles s'y opposent pour des raisons politiques. Il y a au bout du compte une inégalité de l'offre selon les CMS, sans que les usagers puissent «choisir» leur centre.

25. Dans un document interne, ronéotypé, un CMS, après avoir noté « qu'il y a des situations chroniques sur lesquelles on introduit peu ou pas de changement » se demande « s'il est nécessaire, malgré la problématique, de les garder en suivi ». Il répond « que le suivi n'est pas de garder des situations ».
} 
Olivier Cousin

mais leurs organismes de tutelle. Ce n'est pas la sélection qui est contestable, c'est l'arbitraire qui empêche de l'organiser. Le risque ici est le recours insidieux à une classification des pauvres en fonction de leur mérite ce qui conduirait à une autre forme de contrôle social, peut-être plus brutal qu'il ne l'était dans les années 1970, période de dénonciation. ${ }^{26}$

\section{CONCLUSION}

Les expérimentations des centres médicaux sociaux ne se limitent donc pas à une simple adaptation à des contraintes structurelles. Elles engendrent des changements profonds dans le travail social conduisant à redéfinir le public des CMS et à repenser les modes de prise en charge. Ces transformations sont imposées par le contexte et s'imposent si les travailleurs sociaux ne veulent pas perdre leurs positions dans le champ de l'action sociale. Pour s'adapter, les assistantes sociales introduisent de nouvelles pratiques s'appuyant sur des principes d'évaluation et de partenariat. Ces nouvelles méthodes de travail peuvent modifier le lien entre les travailleurs sociaux et les usagers puisqu'ils remettent en cause les principes de la relation et le modèle psycho-éducatif. Plus profondément, c'est le sens du travail social qui se transforme. L'objectif d'un suivi social est moins de corriger des manières d'être que d'offrir des moyens de réinsertion.

Si les CMS, en optant pour des changements d'organisation, deviennent plus efficaces, la recherche ne permet pas de dégager un modèle plus efficient qu'un autre. Les choix s'inscrivent dans un contexte social, géographique et politique particulier et ce qui est pertinent pour la métropole régionale ne l'est en aucun cas pour une circonscription rurale. Par exemple, la quasi-spécialisation autour des personnes isolées rencontre un échos favorable dans les grandes villes, elle n'a pratiquement aucun sens en zone rurale. Mais, par ailleurs, dans le cadre d'une société dominée par les problèmes de l'exclusion et de la pauvreté, ces logiques butent rapidement sur un obstacle : l'absence d'emploi et d'une réelle possibilité d'insertion. C'est pourquoi il est nécessaire de délimiter le champ de l'action sociale afin de ne pas faire reposer sur le travail social ce qui ne relève pas de sa compétence. Le danger serait de rendre les travailleurs sociaux responsables des orientations de la politique sociale. Ils doivent rester les exécutants d'une politique élaborée et définie par le politique au niveau national et local, ce qui ne paraît pas être toujours le cas. La recherche de l'efficacité, la

26. Cf. en particulier le $\mathrm{n}^{\circ}$ de la revue Esprit consacré à la dénonciation du contrôle social dans l'activité des travailleurs sociaux. Esprit, (1984) « Pourquoi le travail social ?», 1ère édition, avril - mai 1972. 
Les mutations du travail social volonté d'évaluer les actions et l'introduction d'un contrat entre les travailleurs sociaux et les usagers doivent donc être relativisées, il ne s'agit que de moyens. Ces notions n'ont qu'une valeur relative au regard de l'action sociale et non pas absolue. Les travailleurs sociaux ne peuvent que préserver le lien social mais leurs actions n'ont qu'un très faible impact sur l'insertion. Comme le souligne R. Castel, « on ne fonde pas la citoyenneté sur de l'inutilité sociale ». ${ }^{27}$ Ici, nous sortons du cadre du travail social.

\section{BIBLIOGRAPHIE}

Ballion R. (1982), Les consommateurs d'école, Paris, Stock.

Castel R. (1995), Les métamorphoses de la question sociales, Paris, Fayard.

Cogant C. et Cousin O. (1995), Evaluation des expérimentations sur la polyvalence de secteur, LAPSAC, Université de Bordeaux II.

Donzelot J.(1991), (sous la direction de), Face à l'exclusion, Paris, Esprit.

Dubet F. (1994), Sociologie de l'expérience, Paris, Le Seuil.

Durrleman A. (mars 1993), (sous la direction de), Commissariat Général au Plan, «Redéfinir le travail social, réorganiser l'action sociale», Préparation du XIème plan, Paris, La Documentation française.

Economie et Statistique (février 1995), n² 277/278.

Esprit (1984), « Pourquoi le travail social ? ». 1ère édition, (avril - mai 1972).

Ion J. (1990), Le travail social à l'épreuve du territoire, Paris, Privat.

Kepel G. (1987), Les banlieues de l'Islam, Paris, Deuil.

Lapeyronnie. D. (1993), L'individu et les minorités, Paris, P.U.F.

Rapport de la Commission Nationale d'évaluation du RMI (1992), Paris, La Documentation française, .

Le RMI à l'épreuve des faits (1991), Paris, Syros.

Rosanvallon P. (1995), La nouvelle question sociale, Paris, Le Seuil.

Verdès-Leroux J. (1978), Le travail social, Paris, Editions de Minuit,

Olivier COUSIN

Centre d'analyse et d'intervention sociologiques-CADIS /URA CNRS 985

Université de Bordeaux II

3 ter, place de la victoire 33076 Bordeaux Cedex

MOTS CLÉS

27. R. Castel, op. cit. p. 429. 
Olivier Cousin

Action sociale, assistance sociale, politique sociale, travailleur social, usager. 